\title{
Wenn der Druck raus ist - die verschiedenen Schockformen
}

Michael Gores, Jennifer Schneck

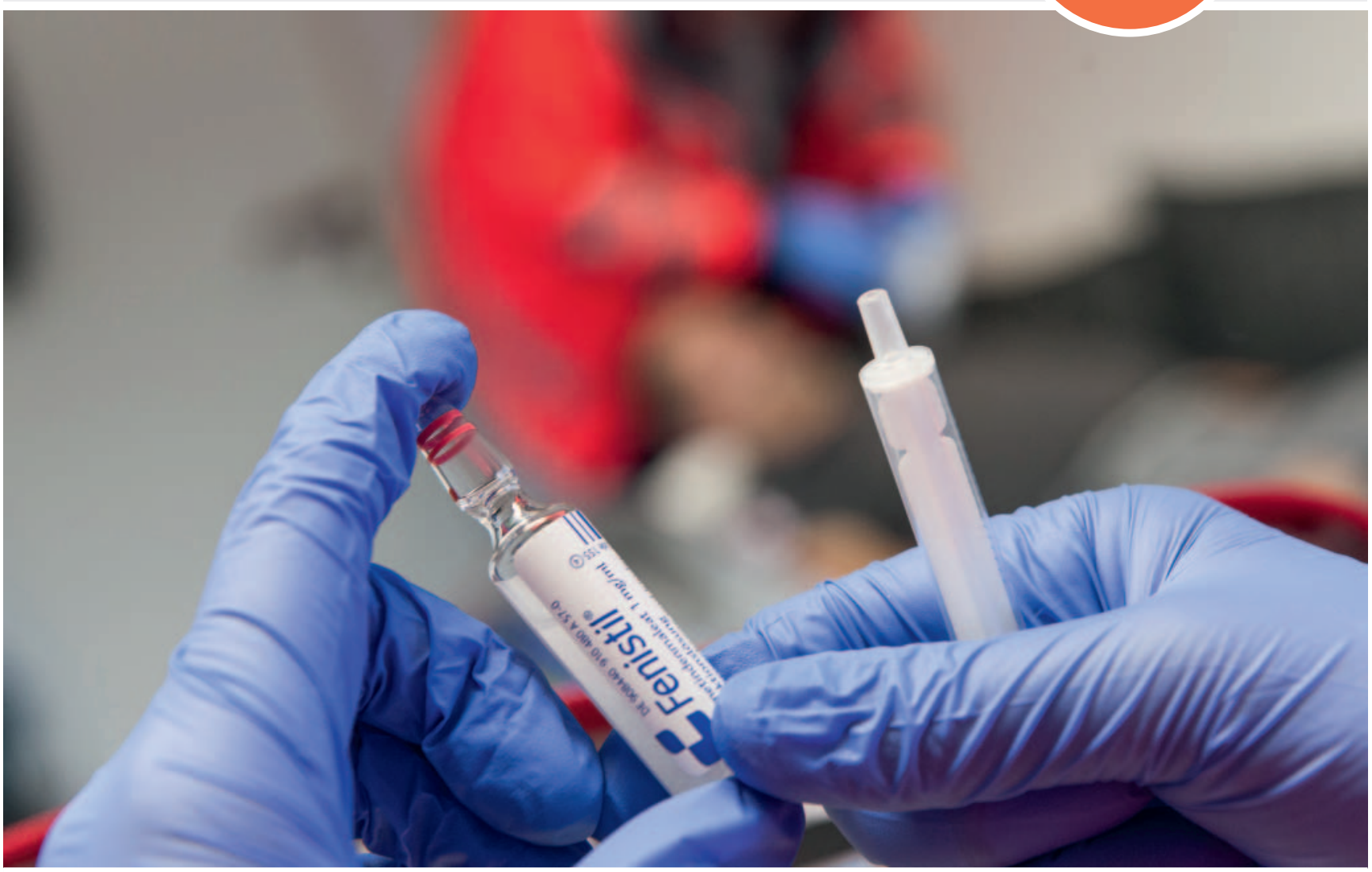

Quelle: KH Krauskopf

Patienten im Schock befinden sich in einem lebensbedrohlichen Zustand. Ein schnelles, zielorientiertes Handeln ist notwendig, um das Überleben zu sichern und um nach einer Behandlung das bestmögliche Endergebnis (Outcome) zu erreichen. In diesem Artikel lernen Sie anhand von Fallbeispielen die verschiedenen Schockformen und ihre Pathophysiologie sowie die Therapie kennen.

\section{Allgemeine Definition von Schock}

Der Schock ist ein medizinischer Notfall, bei dem durch eine schwere Kreislaufstörung die Sauerstoffversorgung des Gewebes im kritischen Maße reduziert ist. Ursache können folgende Krankheitsbilder sein:

- hypovolämischer Schock,

- allergischer (anaphylaktischer) Schock,

- spinaler Schock,

- kardiogener Schock,

- septischer Schock.

\section{Hypovolämischer Schock}

\section{FALLBEISPIEL}

Verkehrsunfall mit Frontalzusammenprall der Fahrzeuge ( $\bullet$ Abb. 1). Die Motorhaube des Kleinwagens ist zusammengedrückt, alle Airbags sind ausgelöst, und das Armaturenbrett sowie das Lenkrad klemmen den Fahrzeugführer ein. Dieser ist nur noch verzögert ansprechbar, und bei der Untersuchung gemäß CABCDE-Schema [1] vermuten Sie ein relevantes Becken- und Oberschenkeltrauma. Zusätzlich fällt 


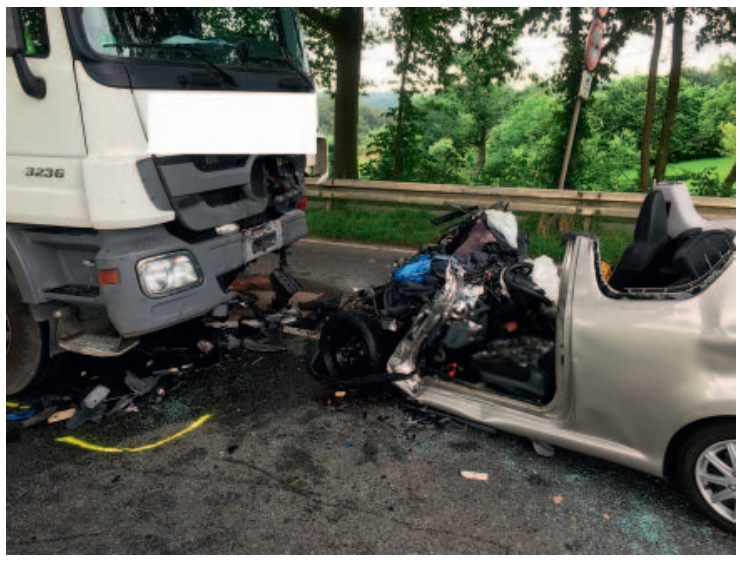

- Abb. 1 Frontalzusammenprall eines Kleinwagens mit einem Lkw.

Ihnen eine starke Blutung am linken Unterschenkel auf. Aufgrund des stark eingedrückten Armaturenbretts ist die Blutungsstelle nicht erreichbar, und Sie entscheiden sich zur Anlage eines Tourniquets.

\section{Definition}

Bei einem Volumenmangelschock ist das intravasale Volumen kritisch reduziert. Dies kann Folge eines hohen Blutverlusts oder anderer Flüssigkeitsverluste bei z. B. Verbrennungen, Durchfall oder Erbrechen sein.

\section{Pathophysiologie}

Geringe Blut-/Flüssigkeitsverluste ( $\triangleright$ Tab. 1) können durch den Organismus kompensiert werden. Sinkt das intravasale Volumen weiter, bewirkt die Ausschüttung körpereigener Katecholamine eine Kontraktion der Blutgefäße, um den Blutdruck aufrechtzuhalten. Dies kann im Extremfall dazu führen, dass nur die lebenswichtigen Organe durchblutet werden (Zentralisation) [2]. Des Weiteren ist das Volumen, das das Herz pro Minute durch den Körper pumpt, entscheidend für die Versorgung der Organe und Gewebe mit Sauerstoff:

Herzzeitvolumen $(H Z V)=$ Schlagvolumen $\times$ Herzfrequenz/min

Bei reduziertem Schlagvolumen kann das gleiche Herzzeitvolumen nur durch eine Erhöhung der Herzfrequenz aufrechterhalten werden.

\section{Therapie}

- Stoppen einer kritischen Blutung gemäß CABCDE (c= critical Bleeding).

- Tourniquet mit Datum und Uhrzeit beschriften.

- Legen eines großlumigen Venenzugangs.
- Tab. 1 Orientierungswerte: Blutverluste und ihre Ursachen.

\begin{tabular}{|l|l|}
\hline Fraktur & Potenzieller Blutverlust \\
\hline Unterarm & bis zu $400 \mathrm{ml}$ \\
\hline Oberarm & bis zu $800 \mathrm{ml}$ \\
\hline Unterschenkel & bis zu $1000 \mathrm{ml}$ \\
\hline Oberschenkel & bis zu $2000 \mathrm{ml}$ \\
\hline Becken & bis zu $4000 \mathrm{ml}$ \\
\hline
\end{tabular}

- Gabe von kristalloiden Vollelektrolytlösungen (z. B. Jonosteril, Sterofundin, Ringer-Lösung).

- „Isotone“ Kochsalzlösung $\mathrm{NaCl}$ 0,9\% vermeiden: Gefahr eines pH-Wert-Abfalls (hyperchlorämische Azidose) mit Minderung der Herzleistung, Gerinnungsstörung und Hemmung der Freisetzung von Noradrenalin [3].

- Kolloidale Infusionslösungen (z. B. HAES $6 \%$ ) sind zurzeit lediglich zugelassen bei Hypovolämie infolge Blutverlusts [4].

- Akzeptanz von Blutdruckwerten von ca. 80 mmHg systolisch sowie einem mittleren arteriellen Druck von ca. $50 \mathrm{mmHg}$ (permissive Hypotension) [5]. Wirkt einem weiteren Blutverlust entgegen.

- Gabe von $1 \mathrm{~g}$ Tranexamsäure zur Förderung der Blutgerinnung. Tranexamsäure wird als Antifibrinolytikum bezeichnet, da es zu einer Hemmung der Gerinnselauflösung (Fibrinolyse) führt [6].

\section{Allergischer (anaphylaktischer) Schock}

\section{FALLBEISPIEL}

Ein 55-jähriger Landwirt mit bekannter allergischer Disposition auf Insektengifte meldet sich bei der Notrufzentrale, da er bei Arbeiten auf dem Feld von einer Wespe gestochen wurde. Sein Notfallset habe er zu Hause vergessen, ihm werde nun zunehmend schwindelig, und eine leichte Luftnot stelle sich ein. Bei Eintreffen des Rettungsteams liegt er kaltschweiBig mit schnellem und schwachem Puls am Boden. Beim Einatmen ist ein pfeifendes Geräusch (Stridor) zu hören.

\section{Definition}

Der allergische (anaphylaktische) Schock ist die Extremform einer allergischen Reaktion, bei der durch Ausfall von wichtigen Körperfunktionen ein lebensbedrohlicher Zustand eintritt. 


\section{Pathophysiologie}

Obwohl allergische Reaktionen bereits vor ca. 2000 Jahren schriftlich erwähnt wurden, ist der Begriff „Allergie“ erst 1906 durch den Kinderarzt Clemens von Pirquet geprägt worden [7].

\section{Einteilung}

Die heutige Einteilung in die 4 Haupttypen (s. Übersicht) wurde 1963 von Coombs und Gell entwickelt [8].

\section{ÜBERSICHT \\ Einteilung des allergischen (anaphylaktischen) Schocks \\ Typ-I-Reaktion (Soforttyp) \\ (s. a. A Abb. 2) \\ - Der Erstkontakt von Körper und Allergenen bleibt symptomlos. \\ - T2-Helferzellen senden Botenstoffe zu B-Lymphozyten $\rightarrow$ Antikörperbildung. \\ - Bindung von Antikörpern auf der Oberfläche von Mastzellen. \\ - Zweitkontakt: Ausschüttung von u. a. Histamin.}

Typ-II-Reaktion (zytotoxischer Typ)

- Medikamente/Bluttransfusionen binden als Antigene an Körperzellen.

- Immunglobuline im Blut erkennen die Antigene und leiten dessen Auflösung (Lyse) ein.

- Eine Unterscheidung zwischen Antigen und eigener Körperzelle ist nicht möglich.

Typ-III-Reaktion (Immunkomplex-Typ)

- Ablagerung von Antigen-Antikörper-Komplexen an Blutgefäßen und Organen.

- Auslösen einer Entzündungsreaktion und evtl. ein Gefäßverschluss, bei dem das nachfolgende Gewebe durch Sauerstoffmangel geschädigt wird.

Typ-IV-Reaktion (Spättyp)

- Symptome treten zeitverzögert erst nach 1 - 2 Tagen auf.

- Keine Vermittlung durch Antikörper.

- T-Lymphozyten und Helferzellen greifen ohne Zuhilfenahme von Immunglobulinen Antigene an.

Die Typ-I-Reaktion (Soforttyp) ist die im Rettungsdienst am häufigsten vorkommende allergische Reaktion.

\section{Histaminrezeptoren}

Histamin fungiert als Mediator und löst eine große Zahl von Folgereaktionen aus. Seine Wirkung vermittelt Histamin über die membrangebundenen Histaminrezeptoren

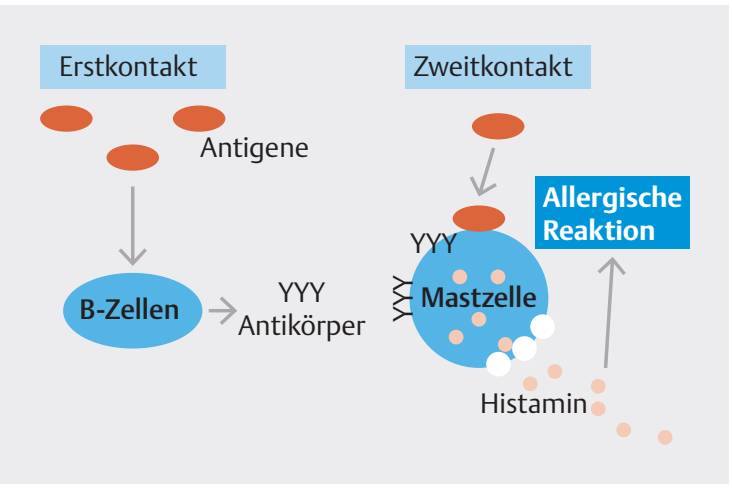

- Abb. 2 Pathophysiologie der Typ-I-Reaktion [9].

$\left(\mathrm{H}_{1}\right.$ bis $\left.\mathrm{H}_{4}\right)$. Bei allergischen Reaktionen sind vor allem $\mathrm{H}_{1}$ - und $\mathrm{H}_{2}$-Rezeptoren beteiligt.

- $\mathrm{H}_{1}$-Rezeptoren:

- Blutgefäße:

- Gefäßweitstellung mit Blutdruckabfall,

- Ödembildung aufgrund erhöhter Durchlässigkeit,

- Schwellung, Hautrötung,

- Bronchien:

- Verengung der Bronchien (Bronchokonstriktion).

- $\mathrm{H}_{2}$-Rezeptoren:

- Magen-Darm-Trakt: vermehrte Bildung von Magensäure.

\section{Therapie}

- Unterbrechung der Allergenzufuhr.

- 1 Venenzugang mit Vollelektrolytlösung.

- Gabe von Adrenalin (Aktivierung von $\alpha$ - und $\beta$-Rezeptoren als Gegenspielern zum Histamin $\rightarrow$ Gefäßengstellung/Bronchienerweiterung/Reduktion von Ödemen).

- 0,3-0,5 mg als intramuskuläre Gabe; ggf. Wiederholung,

- ggf. bei unzureichender Stabilisierung: Spritzenpumpe (Dosierung: 0,05-1 $\mathrm{kg} / \mathrm{kg} \mathrm{KG/min)}$

- Gabe eines $\mathrm{H}_{1}$-Rezeptorantagonisten (Clemastin $0,05 \mathrm{mg} / \mathrm{kg}$ KG oder Dimetinden 0,1 mg/kg KG).

- Gabe eines $\mathrm{H}_{2}$-Rezeptorantagonisten (z. B. Cimetidin $5 \mathrm{mg} / \mathrm{kg} \mathrm{KG}$ ) bei schwerer Anaphylaxie. $\rightarrow$ Ob die Gabe einen Vorteil bringt, ist bis jetzt nicht abschließend geklärt.

- Gabe von Glukokortikoiden (z. B. Prednisolon 1 $2 \mathrm{mg} / \mathrm{kg} \mathrm{KG}$ ). $\rightarrow$ Wichtig, auch wenn die Wirkung deutlich verzögert erst nach 30-60 Minuten eintritt [10].

\section{Spinaler Schock}

\section{FALLBEISPIEL}

Beim Blick aus dem Fenster der Rettungswache sehen Sie eine umgestürzte Leiter und daneben eine Person auf dem Boden liegend. Sie haben die Situa- 
tion gleich erkannt und melden der Leitstelle Ihr Ausrücken zum Einsatzort. Beobachter der Situation schildern, dass die Sturzhöhe ca. $4-5 \mathrm{~m}$ betrug. Ihr Patient ist wach, ansprechbar und orientiert. In Ihrer Untersuchung gemäß CABCDE-Schema ist bei unauffälligen Befunden für Airway und Breathing ein schwacher Puls zu tasten. Keine Einblutung in die großen Blutungsräume. Der Patient gibt an, er könne die Beine nicht mehr bewegen. Trotz der niedrigen Blutdruckwerte ist der Patient nicht kaltschweißig, sondern zeigt eine trockene und überwärmte Haut. Mittels Spineboard wird der Patient immobilisiert und in die nächste Klinik mit neurochirurgischer Abteilung gebracht.

\section{Definition}

Der spinale Schock ist eine Form des neurogenen Schocks, bei der aus dem Ausfall der Regulationsmechanismen auf der Ebene des Rückenmarks ein niedriger Blutdruck resultiert.

\section{Pathophysiologie}

Die Aufrechterhaltung vieler lebenswichtiger Körperfunktionen wird durch das sogenannte autonome/vegetative Nervensystem gesteuert. Dazu zählen vor allem Blutdruck, Atmung und Herzfrequenz. Der Sympathikus als ein Teil dieses Systems erhöht die Leistungs- und Alarmbereitschaft des Körpers. Als sogenannter Gegenspieler wirkt der Parasympathikus. Die einzelnen Einflüsse von Sympathikus und Parasympathikus sind in \ Tab. 2 gegenübergestellt.

Eine traumatische Durchtrennung des Rückenmarks führt zu einer fehlenden nervalen Innervation unterhalb der Läsion [11]. Die fehlende Regulation des Sympathikus an den Blutgefäßen sorgt für eine Gefäßweitstellung, wodurch der Blutdruck deutlich absinkt (relativer Volumenmangel). Im Gegensatz zu anderen Schockarten zeichnet den neurogenen Schock eine warme (Gefäßerweiterung) und trockene (fehlende sympathische Innervation der Schweißdrüsen) Haut aus.

Je nach Höhe der Läsion können auch weitere lebenswichtige Funktionen beeinträchtig sein. Das Herz erhält seine zuführenden Nervenfasern aus dem Bereich der Brustwirbel 1 - 4. Ein Ausfall des Sympathikus sorgt dort für ein Überwiegen des Parasympathikus mit erniedrigter Herzfrequenz (Bradykardie).

Der Zwerchfellnerv (N. phrenicus) entspringt aus einem Nervengeflecht am Hals (Zervikalnerv III-V). Läsionen in diesem Bereich führen zu mechanischen Atemstörungen (Ateminsuffizienz) mit Indikation zur Beatmung. Zusätzlich kommt es unterhalb des Verletzungsniveaus zu
- Tab. 2 Darstellung der Wirkung des autonomen (vegetativen) Nervensystems am Erfolgsorgan.

\begin{tabular}{|l|l|l|}
\hline Erfolgsorgan & Sympathikus & Parasympathikus \\
\hline Herz & Herzfrequenz $\uparrow$ & Herzfrequenz $\downarrow$ \\
\hline Blutgefäße & Verengung & $\begin{array}{l}\text { Gefäßerweiterung (indirekte } \\
\text { Wirkung durch Hemmung } \\
\text { des Sympathikus) }\end{array}$ \\
\hline Lunge & Bronchienerweiterung & Bronchienverengung \\
\hline Magen-Darm-Trakt & Darmbewegung $\uparrow$ & Darmbewegung $\downarrow$ \\
\hline Schweißdrüsen & vermehrtes Schwitzen & keine Wirkung \\
\hline
\end{tabular}

einem inkompletten oder kompletten Ausfall von Empfindungen (Sensibilität) und Beweglichkeit (Motorik).

\section{Therapie}

- Untersuchung gemäß cABCDE-Schema.

- Die Immobilisierung mittels Spineboard oder Vakuummatratze ist unerlässlich.

- 1 Venenzugang legen.

- Volumengabe (Vollelektrolytlösung) zur Therapie der relativen Hypovolämie.

- Gegebenenfalls Noradrenalin bei unzureichender Stabilisierung: Spritzenpumpe (Dosierung: 0,05$1 \mu \mathrm{g} / \mathrm{kg} / \mathrm{min})$.

- Sauerstoffgabe, ggf. Intubation bei mechanischem Atemversagen.

Die Gabe von Methylprednisolon wird nicht mehr empfohlen, da kein verbessertes Endergebnis resultiert [12].

\section{Kardiogener Schock}

\section{FALLBEISPIEL}

Der Gast eines Restaurants klagt nach dem Essen über Schmerzen in Brustkorb und linkem Arm. Im RTW beginnen Sie mit der Basisdiagnostik und sehen ST-Streckenhebungen im EKG. Unter dem Bild eines akuten Herzinfarkts beginnen Sie Ihre Therapie. Zeitnah wird der Patient in die nächstgelegene Klinik mit Herzkatheterlabor gebracht.

Kurz nach Fahrtbeginn zeigt er plötzlich ein blasses kaltschweißiges Hautkolorit. Der Blutdruck ist kaum noch messbar, und der Puls ist schwach und schnell. In der Notfallechokardiografie in der Notaufnahme ist zu sehen, dass die Herzleistung deutlich eingeschränkt ist. Ein Arzt in der Notaufnahme erklärt Ihnen, dass ein kardiogener Schock bei ca. 5-10\% aller Herzinfarkte auftritt und eine hohe Sterblichkeit aufweist. 


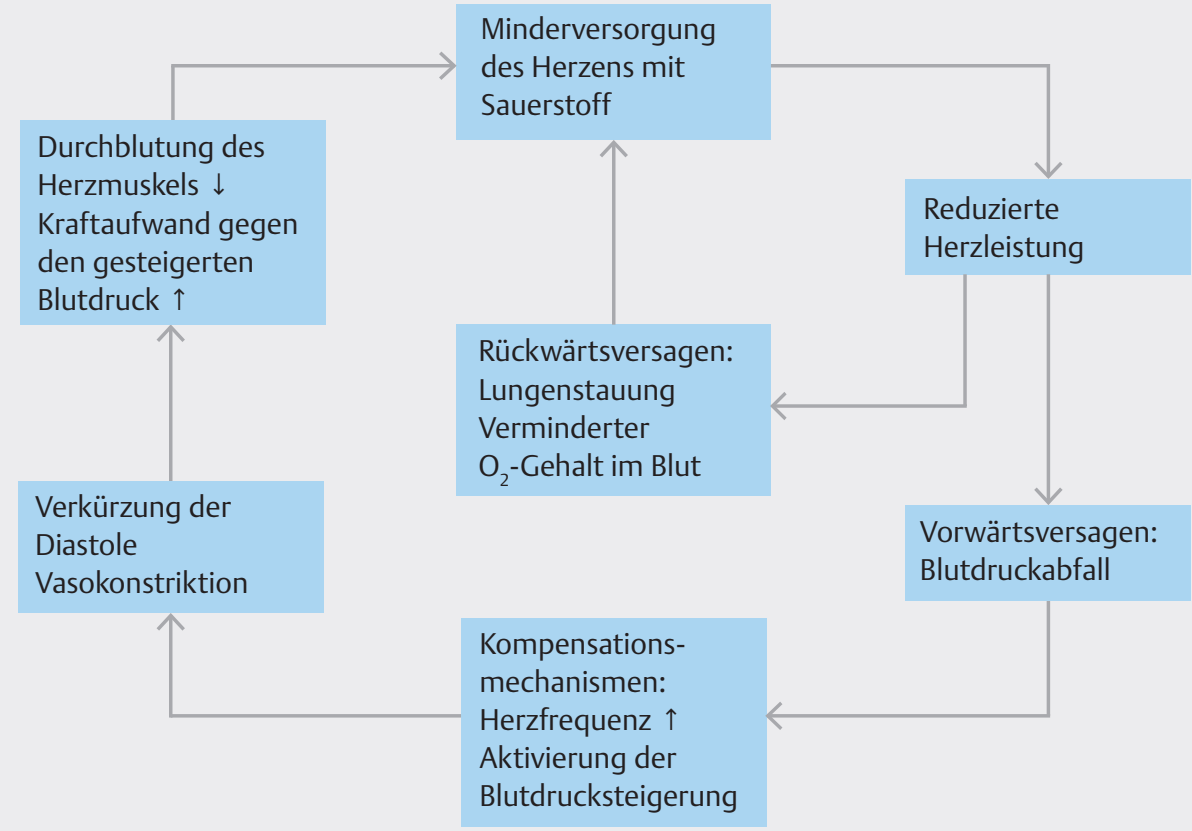

Abb. 3 Circulus vitiosus (Teufelskreis) bei kardiogenem Schock.

\section{Definition}

Bei einem kardiogenen Schock kommt es zu einem Missverhältnis zwischen Sauerstoffbedarf und Angebot im Gewebe. Ursache ist eine Störung der Pumpfunktion des Herzens.

\section{Klinik, Symptomatik}

\section{ÜBERSICHT}

Symptome des kardiogenen Schocks

- niedriger Blutdruck (Hypotonie)

- schneller Puls (Tachykardie)

- bläuliches Hautkolorit (Zyanose)

- kaltschweißige Haut

- gestaute Halsvenen

- Bewusstseinseinschränkung

- geringe Urinausscheidung (Oligurie)

\section{Pathophysiologie}

\section{ÜBERSICHT}

\section{Mögliche Ursachen eines kardiogenen Schocks}

- Herzinfarkt

- Herzmuskelentzündung (Myokarditis)

- akute Herzklappenerkrankungen

- Ruptur eines Ventrikels oder des Septums

- akuter Verschluss des Ausflusstraktes (z. B. Lungenembolie)
Werden die Ursachen eines kardiogenen Schocks nicht zeitnah behoben, kommt es oft zu einer Abwärtsspirale bis hin zum Tod. In einem Teufelskreis (Circulus vitiosus; - Abb. 3) versucht der Körper, durch körpereigene Mechanismen den Kreislauf aufrechtzuerhalten [13]. Diese Mechanismen führen oft zu einer Verschlechterung der Gesamtsituation.

Bei einem Herzinfarkt kommt es zu einem Verschluss eines Herzkranzgefäßes mit einer Sauerstoffunterversorgung des Herzmuskels. Resultiert aus der verschlechterten Herzleistung ein niedriger Blutdruck, versucht der Körper gegenzusteuern, um das Herzminutenvolumen aufrechtzuerhalten. Eine Möglichkeit ist eine erhöhte Herzfrequenz (Tachykardie). Da die Eigenversorgung des Herzens mit Blut in der Entspannungsphase des Herzens (Diastole) stattfindet und die Diastole bei einer höheren Herzfrequenz zeitlich verkürzt wird, resultiert daraus eine verschlechterte Eigenversorgung.

Die Niere detektiert einen zu geringen Blutdruck und bildet ein hormonähnliches Enzym (Renin), das in Wechselwirkung mit anderen Enzymen (Renin-Angiotensin-Aldosteron-System, RAAS) sowohl zu einer starken Gefäßverengung (Vasokonstriktion) als auch zu einer Rückresorption von Flüssigkeit führt [14].

Gegen einen höheren Blutdruck in der Schlagader (Aorta) muss das geschwächte Herz mehr Kraft aufwenden, was einen höheren Sauerstoffbedarf bedeutet. Im Rahmen des Rückwärtsversagens kommt es zum Aufstau von Blut 
quick-SOFA

(Sepsis-Related Organ Failure Assessment)

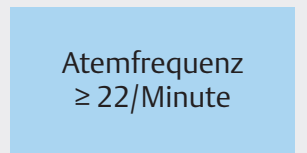

systolischer
Blutdruck
$<100 \mathrm{mmHg}$

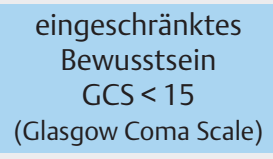

Bei 2 oder mehr Punkten besteht das Risiko eines deutlich schlechteren Endergebnisses

Abb. 4 quickSOFA-Score.

in der Lunge. Das dadurch entstehende Lungenödem sorgt für eine verminderte Aufnahme von Sauerstoff ins Blut.

\section{Therapie}

- Die Behebung der Ursache des kardiogenen Schocks spielt die wichtigste Rolle.

- Zügiger Transport in eine geeignete Klinik.

- Basisdiagnostik mit 12-Kanal-EKG, Blutdruckmessung und Pulsoxymetrie.

- Oberkörperhochlagerung $\left(R_{\text {syst. }}>80 \mathrm{mmHg}\right)$ oder Flachlagerung.

- 1 Venenzugang legen.

- Gegebenenfalls Katecholamingaben:

- Noradrenalin 0,05-1 $\mathrm{gg} / \mathrm{kg} \mathrm{KG} / \mathrm{min}$,

- Dobutamin 2,5 - $10 \mu \mathrm{g} / \mathrm{kg} \mathrm{KG/min.}$

- Einerseits kritisch, da der Sauerstoffverbrauch des Herzens gesteigert wird.

- Andererseits besteht bei unzureichendem Blutdruck/ Auswurfleistung oftmals keine andere Möglichkeit.

\section{Septischer Schock}

\section{FALLBEISPIEL}

Ein RTW-Team wird zu einer älteren Dame (82 Jahre) gerufen und von den Angehörigen in Empfang genommen. Wegen eines Harnwegsinfekts wurde sie mit einem Antibiotikum behandelt. Seit gestern sei sie schläfriger und nun schwer erweckbar. In der Untersuchung zeigt sich die Patientin somnolent (GCS 10), hypoton (RR 92/60 mmHg) und tachypnoeisch (Atemfrequenz $26 / \mathrm{min}$ ). Das Thermometer zeigt $39,3{ }^{\circ} \mathrm{C}$ an. Der Urin ist stark konzentriert und übelriechend. Die Patientin wird in das nächstliegende Krankenhaus mit freier Intensivkapazität gebracht. Der Blutdruck lässt sich zu diesem Zeitpunkt nur noch durch Volumengabe sowie Katecholamine aufrechterhalten.

\section{Definition}

Der Begriff der Sepsis ist im Lauf der Zeit durch drei Konsensuskonferenzen geprägt worden. Neue Erkenntnisse ha- ben im Verlauf dazu geführt, dass die SIRS-Kriterien (s. Infobox „Hintergrundwissen“) im Jahr 2016 durch den qSOFA( $\triangleright$ Abb.4) und SOFA-Score ersetzt wurden. Gründe waren unter anderem, dass die SIRS-Kriterien auch auf gesunde Menschen anwendbar sind (z. B. bei Marathonläufern).

\section{PRAXIS}

Tipp

Der qSOFA-Score (quick Sepsis-related Organ Failure Assessment Score; $\triangleright$ Abb.4) wurde für das schnelle Erkennen einer möglichen Sepsis konzipiert und kann ohne große Hilfsmittel auch präklinisch erhoben werden.

\section{SIRS-KRITERIEN}

(SIRS = Systemic Inflammatory Response Syndrome)

- Leukozyten im Blut $<4000$ oder $>12000 / \mathrm{mm}^{3}$

- Körpertemperatur $\leq 36$ oder $\geq 38^{\circ} \mathrm{C}$

- Herzfrequenz $\geq 90 / \mathrm{min}$

- Atemfrequenz $\geq 20 /$ min oder Hyperventilation mit erniedrigtem $\mathrm{CO}_{2}$

Sepsis

Lebensbedrohliche Organfunktionsstörung (z. B. Lungen-, Nieren-, Leberversagen), hervorgerufen durch eine fehlregulierte Antwort des menschlichen Körpers auf eine Infektion ( $\triangleright$ Abb. 5).

\section{Septischer Schock}

Unterform der Sepsis, bei der ein adäquater Blutdruck nicht durch Infusionen aufrechterhalten werden kann und der Einsatz von Katecholaminen (z. B. Noradrenalin) notwendig ist. Gleichzeitig zeigt das Laktat im Blut einen Wert $>2 \mathrm{mmol} / \mathrm{l}$. 


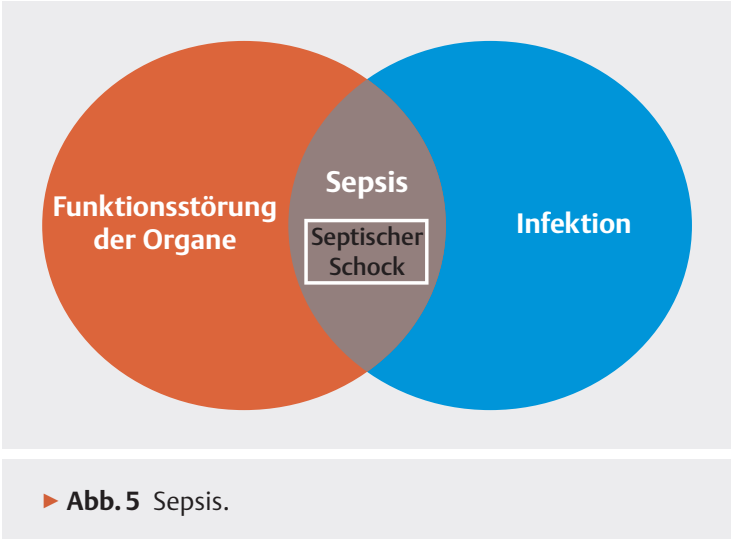

\section{Pathophysiologie}

Im Normalfall ist der Körper in der Lage, kleinere lokale Infektionen selbst einzudämmen und zu behandeln. Nach dem Erkennen von Bakterien wird eine Reihe von Botenstoffen freigesetzt, die unter anderem dafür sorgen, dass lokale Blutgefäße sich weit stellen (Vasodilatation) und die Wände der Gefäße ihre Durchlässigkeit erhöhen. So dringen neben Flüssigkeit auch Abwehrzellen in das Gewebe ein.

Eine entscheidende Rolle in der körpereigenen Abwehr spielt die Antigenpräsentation. Dabei werden den Lymphozyten Bruchstücke des Erregers (Antigen) präsentiert. Die Lymphozyten beginnen daraufhin mit der Bildung auf den Erreger abgestimmter Abwehrstoffe (Antikörper). Gleichzeitig werden im Sinne einer Rückkopplung Botenstoffe zurückgesendet, die verhindern, dass weitere Abwehrzellen rekrutiert werden. Bei Störung der Antigenpräsentation fehlt zum einen die Bildung spezieller Antikörper und zum anderen die Rückkopplung zur Beendigung der Reaktion. Gründe können z. B. Sauerstoffmangel, Trauma, Z. n. Bluttransfusion oder auch eine Chemotherapie sein. Es kommt zu einer Ausbreitung der lokalen Reaktionen auf den ganzen Körper.

Eine generalisierte Weitstellung der Blutgefäße und eine erhöhte Durchlässigkeit sorgen dann für Wassereinlagerungen am ganzen Körper und Blutdruckabfall. Die Minderdurchblutung einzelner Organe kann ein Organversagen hervorrufen oder eine bereits gestörte Funktion weiter verschlechtern [15].

\section{Therapie}

- Zeitnahe Behandlung! Jede Stunde ohne effektive antibiotische Therapie erhöht das Sterberisiko um 7 \% [16].

- 1 Venenzugang legen/Vollelektrolytlösung.

- Stabilisierung der Vitalwerte.

- Gegebenenfalls Noradrenalin bei unzureichender Stabilisierung: Spritzenpumpe (Dosierung: 0,05$1 \mu \mathrm{g} / \mathrm{kg} \mathrm{KG/min).}$

\section{KERNAUSSAGEN}

- Jede Schockform ist ein Notfall und bedarf eines zeitnahen und zielgerichteten Handelns.

- Ist eine Blutung die Ursache eines hypovolämischen Schocks, hat das Stoppen der Blutungsquelle erste Priorität.

- Bei anaphylaktischen Reaktionen wirkt die Gabe von Adrenalin der Histaminwirkung entgegen.

- Die adäquate Immobilisierung von Patienten mit Wirbelsäulentrauma kann verhindern, dass es zu Folgeverletzungen des Rückenmarks und des vegetativen Nervensystems kommt.

- Bleibt die Ursache eines kardiogenen Schocks unbehandelt, kann sich ein Teufelskreis ergeben, der evtl. tödlich endet.

- Mit jeder Stunde einer ausbleibenden Behandlung bei Sepsis steigt die Wahrscheinlichkeit zu versterben.

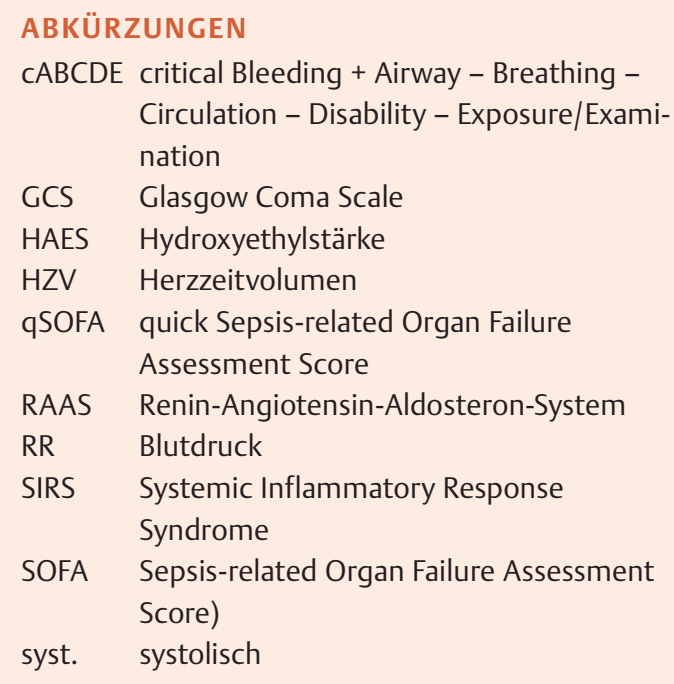

\section{Wissenschaftlich verantwortlich gemäß Zertifizierungsbestimmungen}

Wissenschaftlich verantwortlich gemäß Zertifizierungsbestimmungen für diesen Beitrag ist Michael Gores, Bergisch Gladbach.

\section{Interessenkonflikt}

Die Autoren geben an, dass kein Interessenkonflikt besteht. 


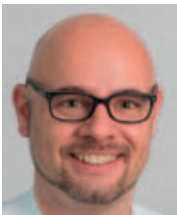

\section{Michael Gores}

Facharzt für Anästhesiologie, Zusatzbezeichnung Intensiv- und Notfallmedizin, Qualifikation LNA/ÄLRD, DEGUM I Anästhesiologie, PHTLS-Provider. Michael Gores ist tätig als Oberarzt in der Abteilung für Anästhesiologie und Intensivmedizin am Evangelischen Krankenhaus Bergisch Gladbach. Zusätzlich ist er Stadtarzt der Malteser in Leverkusen.

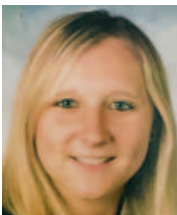

\section{Jennifer Schneck}

2011-2012 RAiP, 2012-2017 Rettungsassistentin. Jennifer Schneck ist Notfallsanitäterin und Praxisanleiterin beim Malteser Hilfsdienst e.V. Leverkusen und seit Oktober 2018 Lehrkraft am Malteser Bildungszentrum Rheinland.

\section{Korrespondenzadresse}

\section{Michael Gores}

Abteilung für Anästhesiologie und Intensivmedizin Evangelisches Krankenhaus Bergisch Gladbach Ferrenbergstraße 24

51465 Bergisch Gladbach

m.gores@evk.de

\section{Literatur}

[1] Maler O, Reich C, Hossfeld B. Taktische Verwundetenversorgung: Großübung des Bundeswehrkrankenhauses Ulm. Rettungsdienst 2015; 8: 783

[2] Adams HA. Schockformen: Hypovolämischer Schock. Anästh Intensivmed 2005; 46: 111-124

[3] Chappell D, Rehm M. Säure-Basen-Haushalt und Balancierte Infusionen- Die Lösung? Makro- Mikrozirkulation Fortbildung Chirur Anästh Intensivmed 2009; 1. Im Internet: https://www. cme-colleg.de/pdf/saeure-basen-haushalt.pdf

[4] Rote-Hand-Brief Hydroxyethylstärke 12.November 2013. Im Internet: https://www.akdae.de/Arzneimittelsicherheit/RHB/ Archiv/2013/20131118.pdf

[5] Kreimeier U, Lackner CK, Prückner S. Permissive Hypotension beim schweren Trauma. Anaesthesist 2002; 10: 787-799
[6] CRASH-2 trial collaborators. Effects of tranexamic acid on death, vascular occlusive events, and blood transfusion in trauma patients with significant haemorrhage (CRASH-2): a randomised, placebo-controlled trial. Lancet 2010; 376: 23-32

[7] Huber B. 100 Jahre Allergie: Clemens von Pirquet - sein Allergiebegriff und das ihm zugrunde liegende Krankheitsverständnis. Wiener Klin Wochensch 2006; 118: 573 579

[8] Heppt W]. Bachert K Praktische Allergologie. Stuttgart: Georg Thieme Verlag; 2010

[9] Sologa J, Klimek L, Knop J et al. Allergologie Handbuch. Grundlagen und klinische Praxis. 2 Aufl. Stuttgart: Georg Thieme/Schattauer; 2011: $79 \mathrm{ff}$

[10] Gesellschaft für Pädiatrische Allergologie und Umweltmedizin e.V. Leitlinien der Gesellschaft für Pädiatrische Allergologie und Umweltmedizin e.V. Akuttherapie und Management der Anaphylaxie (Gültigkeit: bis April 2019). AWMF-LeitlinienRegister-Nummer: 061-025

[11] Schweizer Paraplegiker-Vereinigung. Lehrmittel Paradidact Querschnitt-Medizin und Wissenschaft Zentrale Dienste 2.2012 "Querschnittslähmung“. Im Internet: http://www.spv. ch/__/frontend/handler/document.php?id=245

[12] Bernhard J et al. „Hochdosiertes Methylprednisolon in der Behandlung des akut Querschnittverletzten“. Schweiz Med Forum 2008; 8 (14): $258-259$

[13] Störk S et al. „Akute Herzinsuffizienz und kardiogener Schock Pathophysiologie, Klinik und aktuelle Managementstrategien“. Internist 2005; 46: 285-297

[14] Siegenthaler W et al. Klinische Pathophysiologie. 9 Aufl. Stuttgart: Georg Thieme Verlag; 2006: $684 \mathrm{ff}$

[15] Gerlach H. Die Pathophysiologie der Sepsis. In: Deutsche Akademie für Anästhesiologische Fortbildung (Hrsq). DAAF Refresher Course Aktuelles Wissen für Anästhesisten Nr. 42. Ebelsbach: Aktiv Druck \& Verlag; 2016: 111ff

[16] Kumar A, Roberts D, Wood KE et al. Duration of hypotension before initiation of effective antimicrobial therapy is the critical determinant of survival in human septic shock. Crit Care Med 2006; 34 (6): 1589-1596

Bibliografie

DOI https://doi.org/10.1055/a-0629-0060 retten 2018; 7: 334-343

(c) Georg Thieme Verlag KG, Stuttgart · New York ISSN 2193-2387 


\section{Punkte sammeln auf CEE.thieme.de}

回社回

Diese Fortbildungseinheit ist 12 Monate online für die Teilnahme verfügbar.

Sollten Sie Fragen zur Online-Teilnahme haben, finden Sie unter cme.thieme.de/hilfe

eine ausführliche Anleitung. Wir wünschen viel Erfolg beim Beantworten

der Fragen!

Unter https://eref.thieme.de/CXBZ39V oder über den QR-Code kommen Sie direkt zum Artikel zur Eingabe der Antworten.

\section{Frage 1}

Der Körper versucht, das Herzzeitvolumen zu steigern durch ...

A Steigerung der Atemfrequenz.

B Steigerung der Herzfrequenz.

C Verstärktes Schwitzen.

D Reduktion des Schlagvolumens des Herzens.

E Gefäßweitstellung (Dilatation).

\section{Frage 2}

Welche Zuordnung von Frakturlokalisation und potenziellem Blutverlust ist korrekt?
A Unterarm - bis $5000 \mathrm{ml}$
B Becken - bis $2000 \mathrm{ml}$
C Oberschenkel - bis $1000 \mathrm{ml}$
D Unterschenkel - bis $400 \mathrm{ml}$
E Oberarm - bis $800 \mathrm{ml}$

\section{Frage 3}

Was ist die im Rettungsdienst am häufigsten vorkommende allergische Reaktion?
A Typ-I-Reaktion (Soforttyp)
B Typ-II-Reaktion (zytotoxischer Typ)
C Typ-III-Reaktion (Immunkomplex-Typ)
D Typ-IV-Reaktion (Spättyp)
E Alle Reaktionen kommen gleich häufig vor.

\section{Frage 4}

Was ist keine Wirkung des Sympathikus am Erfolgsorgan?
A Gefäßverengung (Vasokonstriktion)
B Steigerung der Herzfrequenz (Tachykardie)
C vermehrtes Schwitzen (Hyperhidrose)
D Bronchienverengung (Bronchokonstriktion)
E Reduktion der Darmbewegung

\section{Frage 5}

Eine der im Folgenden genannten Maßnahmen gehört zurzeit nicht zum Standardvorgehen bei Patienten mit traumatisch bedingtem spinalem Schock. Welche?
A Immobilisation
B Therapie der relativen Hypovolämie
C Gabe von hochdosiertem Methylprednisolon
D Untersuchung gemäß cABCDE-Schema
E zeitnahe Intubation bei respiratorischer Insuffizienz

\section{Frage 6}

Einer der folgenden Befunde ist kein Symptom des kardiogenen Schocks. Welcher?
A schneller Puls (Tachykardie)
B gestaute Halsvenen
C niedriger Blutdruck (Hypotonie)
D warmes, rosiges Hautkolorit
$\mathrm{E}$ reduziertes Bewusstsein

\section{Frage 7}

Welcher Pathomechanismus beim kardiogenen Schock ist richtig dargestellt?

A Sinkt das Herzzeitvolumen, kommt es kompensatorisch zu einer Bradykardie (Herzfrequenz $\downarrow$ ).

B Bei unzureichendem Blutdruck sorgt die Niere für eine Gefäßerweiterung (Vasodilatation).

C Bei einer Erhöhung der Herzfrequenz kommt es zu einer Verkürzung der Diastole.

D Infolge der Lungenstauung ist mehr Blutvolumen in der Lunge, und die Sauerstoffversorgung wird verbessert.

E Kompensationsmechanismen führen immer zu einer Verbesserung des kardiogenen Schocks.

\section{Frage 8}

Um wie viel Prozent steigt das Mortalitätsrisiko bei septischen Patienten pro Stunde bei fehlender antibiotischer Therapie?
A gar nicht
B $26 \%$
C $3 \%$
D $15 \%$
E $7 \%$

- Weitere Fragen auf der folgenden Seite... 


\section{Punkte sammeln auf CEE.thieme.de}

\section{Frage 9}

Welche Aussage zum quickSOFA-Score ist falsch?

A Das Kriterium „niedrige Atemfrequenz“ wird mit einem Punkt bewertet.

B Der quickSOFA-Score wurde eingeführt, um eine schnelle Beurteilung zu gewährleisten.

C quickSOFA enthält 3 Kriterien zur Beurteilung des Patienten.

D Bei 2 oder mehr Punkten besteht die Gefahr eines verschlechterten Outcomes.

E Der quickSOFA-Score ersetzt die SIRS-Kriterien.

\section{Frage 10}

Eine der folgenden Aussagen ist falsch. Welche?

A SIRS-Kriterien können auch bei gesunden Menschen vorliegen.

B Der septische Schock ist eine Unterform der Sepsis.

C Es gab bis jetzt 3 Konsensuskonferenzen zum Thema Sepsis.

D Aufgrund einer Gefäßweitstellung kommt es bei septischen Patienten zu einer Flüssigkeitsansammlung im Gewebe.

E Bei V. a. septischen Schock ist Noradrenalin kontraindiziert. 